\title{
Monitoring in Metastatic Breast Cancer: Is Imaging Outdated in the Era of Circulating Tumor Cells?
}

\author{
Marianna Alunni-Fabbronia ${ }^{\mathrm{V}}$ Volkmar Müller ${ }^{\mathrm{b}}$ Tanja Fehm ${ }^{c}$ Wolfgang Jannid ${ }^{\mathrm{B}}$ Brigitte Rack $^{\mathrm{a}}$ \\ ${ }^{a}$ Clinic for Gynecology and Obstetrics, Ludwig-Maximilians University, Munich, \\ ${ }^{b} \mathrm{Clinic}$ for Gynecology and Obstetrics, University Hospital Hamburg-Eppendorf, \\ ${ }^{c}$ Clinic for Gynecology and Obstetrics, Heinrich-Heine-University, Düsseldorf, \\ ${ }^{d}$ Clinic for Gynecology and Obstetrics, University Hospital Ulm, Germany
}

\section{Keywords}

Breast cancer - Metastatic breast cancer .

Circulating tumor cells - Minimal residual disease . Imaging $\cdot$ PET/CT $\cdot$ MRI

\section{Summary}

In clinical practice imaging technologies such as computed tomography (CT), positron emission tomography (PET)/CT and magnetic resonance imaging (MRI) are well-established methods for monitoring metastatic breast cancer (MBC) patients and for assessing therapeutic efficacy. However, several weeks of treatment are required before these technologies can offer any reliable information on effective disease regression, and, in the meanwhile, the patients are exposed to potentially unnecessary therapy. Circulating tumor cells (CTCs) have been shown to be powerful prognostic and predictive markers and provide clinicians with valuable information. However, in one clinical trial, an early change of chemotherapy based on CTC detection did not result in improved survival. Currently, CTC detection outside clinical trials should be limited to selected clinical situations, i.e. increased treatment toxicity or as risk estimation.

\section{Introduction}

In women, breast cancer (BC) is the most commonly diagnosed type of cancer, and the risk of developing this disease during life is 1 in 9 [1]. According to the World Health Organization (WHO), BC is the fifth most common cause of death, and alone in 2008, 460,000 lethal cases were registered worldwide [2]. Primary BC detection and diagnosis is mainly based on imaging technologies such as ultrasound and digital mammography $[3,4]$. These methods can help to distinguish between benign and malignant tumors [5], but cannot provide a definitive diagnosis [6]. Despite significant improvements in early diagnosis and treatment, $\mathrm{BC}$ is nevertheless the most common cause of death $(12.7 \%$ of total $)$ from cancer in women worldwide [2]. The high mortality rate is connected to the high tendency of BC to spread, originating metastasis in different organs. Even though no evidence of tumor spread may be seen at the time of the primary diagnosis, a relevant number of axillary node-negative BC patients also develop local or distant metastasis [7, 8]. Bone metastases are frequently diagnosed in $\mathrm{BC}$ patients. In $28-44 \%$ of such cases, they are found at the first relapse as bone-only isolated metastasis [9]. In 50-70\% of the cases they are found in presence of other relapses [10,11], and are responsible for most of the morbidity and disability in metastatic BC (MBC) patients [12]. Over the past few years, many efforts have been made to improve imaging detection to discriminate better between benign and malignant tissues using functional imaging taking into account organ metabolism and local blood flow [13]. Biomedical imaging is commonly involved in all the phases of cancer management, from screening, to prognosis up to therapy, and has the advantage of being minimally invasive and very sensitive [14]. Although imaging has been, and still is, the gold standard in prognosis and metastasis monitoring, there are emerging alternative approaches that could be combined with standard methods to improve the monitoring of the disease status. For example, a growing body of evidence suggests that disseminated tumor cells (DTCs) in bone marrow and circulating tumor cells in peripheral blood (CTCs) might play a key role in the distant spread of the disease. CTCs detach from the primary tumor and, through the blood stream, reach secondary organs where they implant themselves giving origin to micrometastasis. The detection and enumeration of CTCs have been defined as a form of real time 'liquid biopsy' that allows the disease status and development to be monitored in cancer patients $[15,16]$. An early detection of CTCs might have the potential to provide more accurate risk stratification for subsequent therapy decisions, or even help in tailoring additional conventional or targeted therapies to eradicate these

\section{KARGER}

Fax +497614520714

Information@Karger.com

www.karger.com (c) 2014 S. Karger GmbH, Freiburg

$1661-3791 / 14 / 0091-0016 \$ 39.50 / 0$

Accessible online at:

www.karger.com/brc
Dr. Marianna Alunni-Fabbroni

Clinic for Gynecology and Obstetrics

Ludwig-Maximilians University

Maistrasse 11, 80377 Munich, Germany

Marianna.Alunni@med.uni-muenchen.de 
cells before they become overt metastases. In this review, we discuss the different clinical approaches to monitoring tumor status and metastasis development with a special focus on MBC.

\section{Imaging in MBC}

$\mathrm{MBC}$ is an incurable disease and therapy is only palliative with the objective of reducing tumor load, slowing the metastatic progression and increasing the quality of life [17, 18]. Monitoring MBC patients is particularly important during therapy for a prompt identification of patients who do not respond to treatment, which can then be discontinued to avoid unnecessary side effects and to reduce treatment-associated complications such as neutropenia, neuropathy, and alopecia. Serum tumor markers such as CEA and CA 15-3 are widely used in breast cancer management for early and easy evaluation of response or resistance to systemic therapy in patients with advanced disease [19]. However, due to their limited specificity, the use of these markers is often associated with additional detection methods. Biomedical imaging such as ultrasound, positron emission tomography (PET), computed tomography (CT) and magnetic resonance imaging (MRI) are also commonly used in clinical practice. With these methods, the development of the disease can be estimated following the spreading of the metastasis, measuring their size and offering a corresponding evaluation of the response to treatment [2022]. Ultrasound is a ubiquitously available and simple method, widely used for the monitoring of liver metastases in multiple carcinomas. Doppler technologies can help to assess treatment efficacy beyond the mere measurement of tumor size [23]. Several studies have shown a high sensitivity and specificity, comparable to those of CT or MRI [24, 25]. Although the lack of side effects and low costs facilitate repeated measurements, there are limitations to the methodology such as unfavorable conditions, e.g. overweight, and the presence of metastases in locations other than the liver. MRI is used in different clinical contexts, such as in cancer detection, cancer staging and therapy-response monitoring [14]. MRI detects changes in tumor sizes with high sensitivity; however, to observe a significant tumor shrinkage, several cycles of therapy are often necessary. Therefore, MRI is not able to provide early information on treatment efficacy [19]. PET is commonly used in oncology, cardiology and neurology, with oncological applications being the most common [26]. This technology makes use of positron-emitting radioisotopes, most often $\left[{ }^{18} \mathrm{~F}\right]$ fluorodeoxyglucose (FDG), which, after intravenous injection into patients, differentiate malignant from healthy cells [1, 27, 28]. FDG-PET produces 3-dimensional images based on the detection of an altered physiological status of the tissue under examination [5]. In particular, FDGPET monitors the transport, the delivery and the metabolism of glucose - all mechanisms more pronounced in tumor cells than in normal tissues. On the basis of cellular avidity for FDG, data on the type of tumor, on its proliferation, and even on the presence or absence of estrogen receptors are provided. In addition, FDG-PET gives information on MBC staging, as nodal, visceral and bone metastases also usually take up FDG avidly [29, 30]. A comparative study between conventional imaging and FDG-PET showed that the latter is able to predict earlier therapy response, as it can already detect changes in standardized uptake values after the first rounds of chemotherapy [19]. FDG-PET is usually associated with CT, a screening technology making use of an iodinated contrast agent injected intravenously to improve the quality of the anatomical images. FDG-PET/CT scans offer very accurate and precise datasets, combining anatomy inspection and functional analysis for a better monitoring of the tumor status in MBC [31]. The improved sensitivity and specificity of FDG-PET/CT scans makes it possible to discriminate between benign and malignant lesions in $73 \%$ of the cases, and to detect axillary metastasis in $100 \%$ of the cases [5]. By contrast, conventional imaging predicts therapy response on the basis of changes in tumor size, which are usually only evident after 2-3 months of treatment. This is a relevant point in patient handling since an earlier therapy evaluation makes an earlier discontinuation of unnecessary, toxic or ineffective treatments possible.

\section{Circulating Tumor Cells and their Prognostic Value}

Metastases are probably caused by occult hematogenous spreading of tumor cells already during the early phases of the disease. Several studies support the hypothesis that disseminated tumor cells (DTCs) in bone marrow or CTCs in blood of cancer patients, can be regarded as precursors of clinically manifest distant metastases [7, 8, 32-34]. CTCs are tumor cells detached from primary tumors, which may reach distant organs through the bloodstream, and colonize them to form micrometastases $[16,35]$. CTCs can even stay in this status for years before starting to grow further giving rise to macrometastasis $[35,36]$. Secondary metastasis can be initiated if CTCs return to the blood stream and reach other organs. There is evidence to show that CTCs may again reach the primary tumor, starting very aggressive metastatic variants in a process called 'self-seeding' [37, 38]. DTCs can be identified in bone marrow, lymph nodes and blood. In particular, bone marrow appears to be rich in DTCs originating from different sites [39]. However, DTCs can only be isolated from this compartment by needle aspiration, which is a quite difficult and invasive procedure. Moreover, although DTCs have shown good prognostic relevance in early breast cancer, data on their prognostic relevance in metastatic disease are inconsistent, and, therefore, their clinical value unclear [40, 41]. Isolation of CTCs from blood samples is a more attractive option because of its limited invasiveness. Several methods for CTC detection 
and phenotyping, based on immunocytochemical or molecular techniques, are available. The CellSearch ${ }^{\mathrm{TM}}$ system (Veridex, Raritan, NJ, USA) has been validated in the clinical multicenter setting and has proven good reproducibility. It is so far the only method approved by the U.S. Food and Drug Administration to identify and count CTCs in breast, prostate and colon cancer patients. CellSearch ${ }^{\mathrm{TM}}$ is based on magnetic particles, coated with antibodies against the epithelial marker EpCAM, which isolate and enrich the EpCAM-positive CTCs [42]. The cells are then stained with antibodies recognizing CK-8, 18 and 19, and the leukocyte marker CD45, and are then counterstained with DAPI (4,6-diamidino-2-phenylindole) to identify the nucleus. Images, corresponding to the stained cells, are finally displayed in a digital gallery for visual evaluation, with CTCs being defined as $\mathrm{EpCAM}^{+} / \mathrm{CKs}^{+} /$ $\mathrm{DAPI}^{+} / \mathrm{CD}^{-} 5^{-}$cells $[43,44]$. The prognostic relevance of CTC enumeration in MBC patients using CellSearch ${ }^{\mathrm{TM}}$ was demonstrated for the first time by Cristofanilli et al. [45]. The detection of $\geq 5 \mathrm{CTC} / 7.5 \mathrm{ml}$ peripheral blood (PB), before and after a new line of treatment, was associated with a shorter median progression-free survival (PFS) and a shorter overall survival (OS). Therefore, the threshold of $\geq 5 \mathrm{CTC} / 7.5 \mathrm{ml} \mathrm{PB}$ detected with CellSearch ${ }^{\mathrm{TM}}$ has been established for discriminating between a worse and a better prognosis, and variation in the number of CTCs during therapy has been found to be indicative of treatment response or non-response. Moreover, the prognostic role of CTCs associating OS to cell count has been shown to be dependent on the type of primary $\mathrm{BC}$, and is more evident in estrogen receptor-positive and triple-negative MBC than in HER2-positive MBC [46, 47]. However, it should be mentioned that other reports have not confirmed these findings [48, 49]; therefore, this specific issue should be further investigated. The role of CTCs as prognostic markers has been confirmed by many other clinical studies [44, 48, 50 54] and validated by several clinical trials [54-58], all supporting a strong correlation between CTC amount, PFS and OS in MBC. This correlation was also confirmed by a meta-analysis recently presented at the 2013 San Antonio Breast Cancer Symposium [59]. In that investigation, Bidard and coworkers analyzed individual data of 1,944 MBC patients and found that CTC count and changes in their number during therapy were significantly associated with PFS and OS. They concluded that CTC count significantly improves the prognostication of MBC outcome and demonstrated the superiority of CTCs over serum tumor markers.

Although involving early $\mathrm{BC}$ patients and not $\mathrm{MBC}$, it is also worth mentioning the German multicenter SUCCESS trial, which was the largest study so far run to verify the association between CTC enumeration and survival rate [60]. So far, approximately 2,000 early BC patients treated with adjuvant chemotherapy have been monitored with respect to their CTC count by CellSearch ${ }^{\mathrm{TM}}$. In $21.5 \%$ of patients at least 1 CTC was detected before the start of systemic treatment, whereas after completion of chemotherapy, $22 \%$ of patients presented with persisting CTCs. After a median follow-up of 35 months (range 0-54), the presence of CTCs before and after systemic treatment predicted poor disease-free survival (DFS) $(\mathrm{p}=0.0001$ and $\mathrm{p}=0.04)$ and OS $(\mathrm{p}=0.0002$ and $\mathrm{p}=0.03$ ). The prognostic relevance of CTCs in early BC was also confirmed by a recent pooled analysis on 3,172 patients, in which the presence of CTCs was significantly related to shorter PFS ( $p<0.001$, hazard ratio (HR) 2.02, 95\% confidence interval (CI) 1.63-2.50) and OS ( $\mathrm{p}<0.001$, HR 2.57, 95\% CI 1.96-3.37).

\section{Comparison between Imaging and CTCs as Predicting Factors in MBC}

The clinical findings correlating the CellSearch ${ }^{\mathrm{TM}}$-based detection of $\geq 5$ CTCs/7.5 ml PB to PFS and OS both before and 4 weeks after the start of a new line of chemotherapy in MBC opened to the possibility to use CTCs as new prognostic and predictive markers. However, to verify if the number of CTCs correlates with standard imaging determination of disease progression, several validation studies have been conducted. In 2006, the first direct comparison between CTC count and radiological imaging in MBC patients $(\mathrm{n}=138)$ was published [61]. Before starting the therapy, all patients had CT and/or MRI of chest and abdomen, a whole body scan and CTC count by CellSearch ${ }^{\mathrm{TM}}$. Disease status was then monitored every 9 and 12 weeks depending of treatment, using the same clinical and imaging modalities. While both methods could equally predict PFS and OS for patients receiving the first-line treatment, CTCs allowed an earlier and more accurate OS assessment in higher lines of treatment $(\mathrm{p}=0.0009$ for CTCs, $p=0.2209$ for radiological response). Furthermore, the reproducibility of CTC counts was increased compared to radiological assessment with an inter-reader variability of 0.7 and $15.2 \%$, respectively. This study showed for the first time that CTC enumeration is a reliable and accurate way to monitor disease progression, offering an earlier and more reproducible MBC monitoring than standard anatomic imaging methods.

The prognostic significance of CTC enumeration by CellSearch $^{\mathrm{TM}}$ and FDG-PET/CT metabolic imaging was further evaluated in a retrospective analysis of MBC patients $(\mathrm{n}=$ 115) during systemic treatment with standard therapies [62]. CTC counting and PET/CT imaging were performed before starting therapy and monitoring was repeated at the midpoint of therapy (9-12 weeks). The authors found a concordant number of CTCs to FDG-PET/CT response/non-response in $68(67 \%)$ patients and a concordant number of CTCs to FDGPET/CT progression/non-progression in 76 (75\%) patients. In the 34 response/non-response discordant cases (33\%), 31 patients had $\leq 5 \mathrm{CTCs} / 7.5 \mathrm{ml}(91 \%)$ and only $3(9 \%)$ showed $\geq 5 \mathrm{CTCs} / 7.5 \mathrm{ml} \mathrm{PB}$ at therapy midpoint. In the 26 progression/non-progression discordant cases $(25 \%), 22(85 \%)$ 
patients had $\leq 5 \mathrm{CTCs} / 7.5 \mathrm{ml} \mathrm{PB}$, while $4(15 \%)$ showed $\geq 5$ CTCs $/ 7.5 \mathrm{ml} \mathrm{PB}$ at therapy midpoint. The authors suggested a relationship between CTC counts and tumor metabolic activity. Moreover, they found that both FDG-PET/CT response/non-response and FDG-PET/CT progression/nonprogression can reliably predict prognosis in those patients for whom the CTC number is $\leq 5 \mathrm{CTCs} / 7.5 \mathrm{ml} \mathrm{PB}$ at mid-therapy ( $\mathrm{p}=0.0086$ and $\mathrm{p}=0.0018$, respectively), while detection of $\geq 5 \mathrm{CTCs} / 7.5 \mathrm{ml} \mathrm{PB}$ during therapy can reliably monitor disease progression in $\mathrm{MBC}$ patients.

In the same year, a prospective longitudinal clinical study was conducted to validate the hypothesis that the number of CTCs, detected with CellSearch ${ }^{\mathrm{TM}}$, and the fixed threshold of $5 \mathrm{CTCs} / 7.5 \mathrm{ml} \mathrm{PB}$ correlates with the imaging determination of disease progression in MBC patients $(n=81)$ [63]. Before starting a new line of therapy, PB samples were collected and analyzed, and a complete imaging evaluation was performed. PB samples collection and imaging monitoring were repeated in intervals of 9 and 12 weeks, corresponding to new treatment cycles. This study showed that CTC enumeration was strongly correlated to radiographic determination of disease progression in patients receiving treatment for MBC. Importantly, correlation was applicable not only to the time of imaging, but also to CTC results obtained up to 9 weeks in advance with respect to imaging. Moreover, the number of CTCs with respect to the threshold of $5 \mathrm{CTCs} / 7.5 \mathrm{ml} \mathrm{PB}$ was a reliable marker of PFS in the patient cohort. In conclusion, the results of this study supported the importance of CTC quantification and its role in monitoring the disease status of $\mathrm{MBC}$ in combination to the standard imaging methods. FDG-PET/CT has been shown to be a very sensitive method with respect to conventional imaging approaches for detecting MBC metastasis, because of the combination of anatomic and metabolic datasets. To understand if there is a correlation between the number of CTCs and the metabolic activity of metastasis in MBC patients, a new study was conducted by De Giorgi et al. [64] to correlate CTCs and bone metastasis detected by FDG$\mathrm{PET} / \mathrm{CT}$ in patients $(\mathrm{n}=195)$ diagnosed with relapsed/progressive MBC. Before being enrolled in the study, all patients were diagnosed with relapsed/progressive MBC according to standard clinical and imaging procedures. At the beginning of the study, patients underwent FDG-PET/CT and provided PB samples for CTC enumeration by CellSearch ${ }^{\mathrm{TM}}$. In the patient cohort, 103 (53\%) showed $\leq 5 \mathrm{CTCs} / 7.5 \mathrm{ml} \mathrm{PB}$ at relapse/progression and $92(47 \%) \geq 5 \mathrm{CTCs} / 7.5 \mathrm{ml} \mathrm{PB}$. In the group with $\geq 5 \mathrm{CTCs} / 7.5 \mathrm{ml} \mathrm{PB}, 83$ patients $(90 \%)$ showed bone metastasis; in the group of 137 patients with bone metastasis at relapse/progression, $83(61 \%)$ had $\geq 5$ CTCs/7.5 ml PB and 54 $(39 \%)$ had $\leq 5$ CTCs $/ 7.5 \mathrm{ml}$ PB. The authors described a good correlation between the number of CTCs found in patients and the presence of bone metastasis detected by FDG-PET/ CT, with higher numbers of CTCs detected in patients with more extensive bone metastasis. Among patients with no bone metastasis detected by FDG-PET/CT, lower CTC num- bers were detected in case of lymph node and/or chest wall metastasis ( $\mathrm{n}=23$; mean $1.4 \pm 2.0$ CTCs), compared to lung and /or pleural metastases $(\mathrm{n}=25$; mean $2.9 \pm 8.1 \mathrm{CTCs})$ and liver metastasis $(n=9$; mean $8.9 \pm 16.4$ CTCs $)$. CTCs enumeration did not predict OS in patients without metastasis $(\mathrm{p}=$ $0.4111)$ and in those with only bone metastasis $(\mathrm{p}=0.3552)$, while CTCs predicted OS in patients who presented bone metastasis associated with metastasis also in other sites ( $\mathrm{p}=$ 0.0008). In summary, the study correlated the number of CTCs detected in MBC patients and the site of metastasis, with significantly higher CTC numbers if bone metastasis were present. Among patients with bone metastasis, a clear difference in CTC count was observed between patients with diffuse skeletal metastasis and those with few bone lesions. Among patients with metastasis in other tissues, CTC numbers were higher in case of liver metastasis with respect to soft tissues, lung and/or pleural metastasis. These observations suggest that the entity of the tumor shedding, and therefore the number of detected CTCs, might be connected to the site of metastasis, and that a higher number of CTCs might indicate bone involvement. The same authors expanded the study evaluating the predictive significance of CTC enumeration and FDG-PET/CT in patients with only bone metastasis derived from $\mathrm{BC}$ and without any visceral metastasis $(\mathrm{n}=55)$ [65]. All patients underwent FDG-PET/CT and CTC counting by CellSearch ${ }^{\mathrm{TM}}$ before starting a new line of standard treatment. Disease status was further evaluated after 2 and 4 months from the beginning of the study. 43 (78\%) patients showed concordant FDG-PET/CT and CTC results. Among the 12 patients with discordant results, $8(66 \%)$ were found with $\geq 5 \mathrm{CTCs} / 7.5 \mathrm{ml} \mathrm{PB}$ with no evidence of progressive disease identified by FDG-PET/CT, while 4 (33\%) were found with $\leq 5 \mathrm{CTCs} / 7.5 \mathrm{ml} \mathrm{PB}$ and evidence of progressive disease. At follow-up, CTC counts and FDG-PET/CT monitoring were significantly associated with PFS ( $p=0.02$ and $p<0.0001$, respectively) and to OS $(p=0.02$ and $p=0.01$ respectively). However, in multivariate analysis, FDG-PET/CT was the only predictive factor for PFS $(p<0.0001)$, while estrogen receptor status was the only predictive factor for OS $(\mathrm{p}=0.01)$.

To our knowledge, it has not been shown so far that an early change of chemotherapy can improve OS. In particular, using imaging techniques to assess tumor response, it has not been proven that a second line of treatment can improve OS in patients not responding to the first line of chemotherapy. In this respect, Smerage et al. [66] recently presented the results of the SWOG S0500 trial, evaluating a CTC-guided change of chemotherapy regimen in MBC. Patients with elevated CTCs at baseline and also elevated CTCs after 21 days of therapy were randomly assigned to either continue initial therapy until clinical progression or to change to a second-line chemotherapy of the physician's choice immediately at cycle 2.123 patients were randomly assigned to either maintain original chemotherapy $(n=64)$ or switched to new chemotherapy 
$(\mathrm{n}=59)$. Changing to an alternative chemotherapy did not improve OS for patients whose CTCs did not decrease after the first cycle of chemotherapy $(\mathrm{p}=0.83)$. Furthermore, this group of patients with persistently high CTC counts had a relatively short median OS (13 months) compared to patients with initially $\leq 5 \mathrm{CTCs} / 7.5 \mathrm{ml} \mathrm{PB}$ (35 months) or CTCs that dropped $\leq 5 \mathrm{CTCs} / 7.5 \mathrm{ml} \mathrm{PB}$ after the first cycle of chemotherapy (23 months). CTCs might therefore detect a subgroup of patients with resistance to chemotherapy and the need for other treatment approaches.

\section{Conclusions}

The vast literature published during the last few years strongly supports the important role of CTCs in metastasis formation. While the prognostic role of CTCs has been clearly shown for early BC and MBC, the optimal role of CTCs in everyday clinical care is still unclear. In advanced disease, CTCs could predict response to chemotherapy and endocrine treatment effectively, and earlier than imaging tools, and can provide information going beyond mere tumor load. The prognostic information is of potential value, e.g. for the identification of patients with a high risk of rapid tumor progression and the need for chemotherapy. However, a recent trial has shown that early change to an alternative chemotherapy could not improve the prognosis of these patients despite the prognostic relevance of CTCs. Since chemotherapy treatment is usually associated with a number of serious side effects, CTCs could help to avoid unnecessary toxicity when the expected treatment efficacy is low. Additionally, CTC phenotyping could contribute to a better individualized systemic treatment. Changes in the tumor phenotype are a known phenomenon and may lead to treatment changes in up to $20 \%$ of patients. Consequently, CTC phenotyping could be used as an easily accessible, real-time, liquid biopsy of tumor cells that disseminate from the tumor into the blood stream. Clinical intervention trials such as DETECT III are currently being performed to evaluate the predictive role of CTCs to tailor targeted treatment approaches in metastatic diseases. In conclusion, CTC detection and phenotyping offers valuable information on tumor load and tumor biology, and has been shown to be a valid complementary prognostic method to standard imaging. Nevertheless, additional studies and clinical trials will be necessary to establish further applications of CTCs in everyday clinical care.

\section{Disclosure Statement}

M.A-F. and T.F declare no conflicts of interest. V.M. has received speaker honoraria from Amgen, Celgene, Pierre-Fabre, Roche and research funding from Roche. B.R. and W.J. have received educational grants and honorary from Veridex/Johnson and Johnson.

\section{References}

1 Glendenning J, Cook G: Imaging breast cancer bone metastases: Current status and future directions. Semin Nucl Med 2013;43:317-323.

$\checkmark 2$ Ferlay J, Shin HR, Bray F, et al.: Estimates of worldwide burden of cancer in 2008: GLOBOCAN 2008. Int J Cancer 2010;127:2893-2917.

\3 Sree SV, Ng EY, Acharya RU, Faust O: Breast imaging: A survey. World J Clin Oncol 2011;2:171178.

4 Hall FM: Transition to digital mammography. Radiology 2012;262:374

5 De Abreu FB, Wells WA, Tsongalis GJ: The emerging role of the molecular diagnostics laboratory in breast cancer personalized medicine. Am J Pathol 2013;183:1075-1083.

6 Jalalian A, Mashohor SB, Mahmud HR, et al.: Computer-aided detection/diagnosis of breast cancer in mammography and ultrasound: A review. Clin Imaging 2013;37:420-426.

7 Cote RJ, Rosen PP, Lesser ML, et al.: Prediction of early relapse in patients with operable breast cancer by detection of occult bone marrow micrometastases. J Clin Oncol 1991;9:1749-1756.

8 Diel IJ, Kaufmann M, Costa SD, et al.: Micrometastatic breast cancer cells in bone marrow at primary surgery: prognostic value in comparison with nodal status. J Natl Cancer Inst 1996;88:1652-1658.

9 Wei S, Li Y, Siegal GP, Hameed O: Breast carcinomas with isolated bone metastases have different hormone receptor expression profiles than those with metastases to other sites or multiple organs. Ann Diagn Pathol 2011;15:79-83.
10 Jung SY, Rosenzweig M, Sereika SM, et al.: Factors associated with mortality after breast cancer metastasis. Cancer Causes Control 2012; 23:103-112.

11 Manders K, van de Poll-Franse LV, Creemers GJ, et al.: Clinical management of women with metastatic breast cancer: A descriptive study according to age group. BMC Cancer 2006;6:179.

12 Tateishi U, Gamez C, Dawood S, et al.: Bone metastases in patients with metastatic breast cancer: Morphologic and metabolic monitoring of response to systemic therapy with integrated PET/ CT. Radiology 2008:247:189-196.

13 Grizzle WE, Srivastava S, Manne U: Translational pathology of neoplasia. Cancer Biomark 2010;9:720.

14 Fass L: Imaging and cancer: A review. Mol Oncol 2008;2:115-152.

15 Alix-Panabières C, Pantel K: Circulating tumor cells: Liquid biopsy of cancer. Clin Chem 2013;59: 110-118.

16 Alix-Panabières C, Schwarzenbach H, Pantel K: Circulating tumor cells and circulating tumor DNA. Annu Rev Med 2012;63:199-215.

17 Lin NU, Thomssen C, Cardoso F, et al.: International guidelines for management of metastatic breast cancer (MBC) from the European School of Oncology (ESO)-MBC Task Force: Surveillance, staging, and evaluation of patients with early-stage and metastatic breast cancer. Breast 2013;22:203210.
18 Cardoso F, Harbeck N, Fallowfield L, et al.: Locally recurrent or metastatic breast cancer: ESMO Clinical Practice Guidelines for diagnosis, treatment and follow-up. Ann Oncol. 2012;23 Suppl 7:vii11-9.

19 Duffy MJ: Serum tumor markers in breast cancer: Are they of clinical value? Clin Chem 2006;52:345351.

20 Dose Schwarz J, Bader M, Jenicke L, et al.: Early prediction of response to chemotherapy in metastatic breast cancer using sequential 18F-FDG PET. J Nucl Med 2005;46:1144-1150.

21 Therasse P, Arbuck SG, Eisenhauer EA, et al.: New guidelines to evaluate the response to treatment in solid tumors. J Natl Cancer Inst 2000; 92:205-216.

22 Eisenhauer EA, Therasse P, Bogaerts J, et al.: New response evaluation criteria in solid tumours: Revised RECIST guideline (version 1.1). Eur J Cancer 2009;45:228-247.

23 Lagalla R, Caruso G, Finazzo M: Monitoring treatment response with color and power Doppler. Eur J Radiol 1998;Suppl 2:S149-156.

24 Westwood M, Joore M, Grutters J, et al.: Contrastenhanced ultrasound using SonoVue ${ }^{\circledR}$ (sulphur hexafluoride microbubbles) compared with contrast-enhanced computed tomography and contrast-enhanced magnetic resonance imaging for the characterisation of focal liver lesions and detection of liver metastases: A systematic review and costeffectiveness analysis. Health Technol Assess 2013;17:1-243. 
25 Guang Y, Xie L, Ding H, et al.: Diagnosis value of focal liver lesions with SonoVue ${ }^{\circledR}$-enhanced ultrasound compared with contrast-enhanced computed tomography and contrast-enhanced MRI: A metaanalysis. Cancer Res Clin Oncol 2011;137:15951605.

26 Yu JQ, Cristofanilli M: Circulating tumor cells and PET. J Nucl Med 2011;52:1501-1504.

-27 Bos R, van Der Hoeven JJ, van Der Wall E, et al. Biologic correlates of (18F)fluorodeoxyglucose uptake in human breast cancer measured by positron emission tomography. J Clin Oncol 2002;20:379387.

28 Kilburn-Toppin F, Barter SJ: New horizons in breast imaging. Clin Oncol (R Coll Radiol) 2013; 25:93-100.

29 Rosé C, Dose J, Avril N: Positron emission tomography for the diagnosis of breast cancer. Nuc Med Commun 2002;23:613-618.

30 Rohren EM, Turkington TG, Coleman RE: Clinical applications of PET in oncology. Radiology 2004;231:305-332.

31 Cohade C: Altered biodistribution on FDG-PET with emphasis on brown fat and insulin effect. Semin Nucl Med 2010;40:283-293.

-32 Braun S, Pantel K, Müller P, et al.: Cytokeratinpositive cells in the bone marrow and survival of patients with stage I, II, or III breast cancer. N Engl J Med 2000;342:525-533.

33 Schindlbeck C, Pfab G, Jueckstock J, et al.: Prognostic relevance of disseminated tumor cells in the bone marrow of patients with primary breast cancer - results of a standardized follow-up. Anticancer Res 2011;31:2749-2755.

34 Schindlbeck C, Andergassen U, Hofmann S, et al. Comparison of circulating tumor cells (CTC) in peripheral blood and disseminated tumor cells in the bone marrow (DTC-BM) of breast cancer patients. J Cancer Res Clin Oncol 2013;139:10551062.

35 Pantel K, Alix-Panabières C, Riethdorf S: Cancer micrometastases. Nat Rev Clin Oncol 2009;6:339351.

36 Aguirre-Ghiso JA: Models, mechanisms and clinical evidence for cancer dormancy. Nat Rev Cancer 2007;7:834-846.

37 Kim MY, Oskarsson T, Acharyya S, et al.: Tumor self-seeding by circulating cancer cells. Cell 2009; 139:1315-1326.

38 Aguirre-Ghiso JA: On the theory of tumor selfseeding: Implications for metastasis progression in humans. Breast Cancer Res 2010;12:304.

39 Pantel K, Brakenhoff RH: Dissecting the metastatic cascade. Nat Rev Cancer 2004;4:448-445.

40 Bidard FC, Vincent-Salomon A, Sigal-Zafrani B, et al.: Time to metastatic relapse and breast cancer cells dissemination in bone marrow at metastatic relapse. Clin Exp Metastasis 2008;25:871-875.

41 Janni W, Gastroph S, Hepp F, et al.: Prognostic significance of an increased number of micrometa- static tumor cells in the bone marrow of patients with first recurrence of breast carcinoma. Cancer 2000;88:2252-2259.

42 Osta WA, Chen Y, Mikhitarian K, et al.: EpCAM is overexpressed in breast cancer and is a potential target for breast cancer gene therapy. Cancer Res 2004:64:5818-5824.

43 Gorges TM, Pantel K: Circulating tumor cells as therapy-related biomarkers in cancer patients. Cancer Immunol Immunother 2013;62:931-939.

44 Riethdorf S, Fritsche H, Müller V, et al.: Detection of circulating tumor cells in peripheral blood of patients with metastatic breast cancer: A validation study of the CellSearch system. Clin Cancer Res 2007;13:920-928.

45 Cristofanilli M, Budd GT, Ellis MJ, et al.: Circulating tumor cells, disease progression, and survival in metastatic breast cancer. N Engl J Med 2004;351: 781-791

46 Aktas B, Müller V, Tewes M, et al.: Comparison of estrogen and progesterone receptor status of circulating tumor cells and the primary tumor in metastatic breast cancer patients. Gynecol Oncol 2011; 122:356-360

47 Giordano A, Gao H, Anfossi S, et al.: Epithelialmesenchymal transition and stem cell markers in patients with HER2-positive metastatic breast cancer. Mol Cancer Ther 2012;11:2526-2534.

48 Wallwiener M, Hartkopf AD, Baccelli I, et al.: The prognostic impact of circulating tumor cells in subtypes of metastatic breast cancer. Breast Cancer Res Treat 2013;137:503-510.

49 Pierga JY, Hajage D, Bachelot T, et al.: High independent prognostic and predictive value of circulating tumor cells compared with serum tumor markers in a large prospective trial in first-line chemotherapy for metastatic breast cancer patients. Ann Oncol. 2012;23:618-624.

50 Cristofanilli M, Hayes DF, Budd GT, et al.: Circulating tumor cells: A novel prognostic factor for newly diagnosed metastatic breast cancer. J Clin Oncol 2005;23:1420-1430

51 Hayes DF, Cristofanilli M, Budd GT, et al.: Circulating tumor cells at each follow-up time point during therapy of metastatic breast cancer patients predict progression-free and overall survival. Clin Cancer Res 2006;12:4218-4224.

52 Nolé F, Munzone E, Zorzino L, et al.: Variation of circulating tumor cell levels during treatment of metastatic breast cancer: prognostic and therapeutic implications. Ann Oncol 2008;19:891-897.

53 Janni W, Rack B, Terstappen L, et al.: A pooled analysis of the prognostic relevance of circulating tumor cells in early breast cancer, San Antonio Breast Cancer Symposium 2013 (San Antonio, TX, USA)

54 Dawood S, Broglio K, Valero V, et al.: Circulating tumor cells in metastatic breast cancer: From prognostic stratification to modification of the staging system? Cancer 2008:113:2422-2430.
5 Müller V, Riethdorf S, Rack B, et al.: Prognostic impact of circulating tumor cells assessed with the CellSearch System ${ }^{\mathrm{TM}}$ and AdnaTest Breast ${ }^{\mathrm{TM}}$ in metastatic breast cancer patients: The DETECT study. Breast Cancer Res 2012;14:R118.

56 Nakamura S, Yagata H, Ohno S, et al.: Multicenter study evaluating circulating tumor cells as a surrogate for response to treatment and overall survival in metastatic breast cancer. Breast Cancer 2010;17:199-204.

57 Jiang ZF, Cristofanilli M, Shao ZM, et al.: Circulating tumor cells predict progression-free and overall survival in Chinese patients with metastatic breast cancer, HER2-positive or triple-negative (CBCSG004): A multicenter, double-blind, prospective trial. Ann Oncol 2013;24:2766-2772.

58 Bidard FC, Fehm T, Ignatiadis M, et al.: Clinical application of circulating tumor cells in breast cancer: overview of the current interventional trials. Cancer Metastasis Rev 2013;32:179-188.

59 Bidard F-C, Peeters D, Fehm T, et al: Pooled analysis of circulating tumor cells in metastatic breast cancer: Findings from 1944 individual patients data. San Antonio Breast Cancer Symposium 2013 (San Antonio, TX, USA).

60 Rack B, Schindlbeck C, Schneeweiss A, et al.: Prognostic relevance of circulating tumor cells (CTCs) in peripheral blood of breast cancer patients before and after adjuvant chemotherapy The German SUCCESS-Trial. J Clin Oncol (Meeting Abstracts) 2008;26 Suppl:503.

61 Budd GT, Cristofanilli M, Ellis MJ, et al.: Circulating tumor cells versus imaging - predicting overall survival in metastatic breast cancer. Clin Cancer Res 2006;12:6403-6409.

62 De Giorgi U, Valero V, Rohren E, et al.: Circulating tumor cells and $\left[{ }^{18} \mathrm{~F}\right]$ fluorodeoxyglucose positron emission tomography/computed tomography for outcome prediction in metastatic breast cancer. J Clin Oncol 2009;27:3303-3311.

63 Liu MC, Shields PG, Warren RD, et al.: Circulating tumor cells: a useful predictor of treatment efficacy in metastatic breast cancer. J Clin Oncol 2009;27:5153-5159.

64 De Giorgi U, Valero V, Rohren E, et al.: Circulating tumor cells and bone metastases as detected by FDG-PET/CT in patients with metastatic breast cancer. Ann Oncol 2010;21:33-39.

65 De Giorgi U, Mego M, Rohren EM, et al.: 18FFDG PET/CT findings and circulating tumor cell counts in the monitoring of systemic therapies for bone metastases from breast cancer. J Nucl Med 2010;51:1213-1218.

66 Smerage JB, Barlow WE, Hayes DF, et al.: SWOG S0500 - A randomized phase III trial to test the strategy of changing therapy versus maintaining therapy for metastatic breast cancer patients who have elevated circulating tumor cell (CTC) levels at first follow-up assessment. San Antonio Breast Cancer Symposium 2013 (San Antonio, TX, USA). 